\title{
Thelper cell subsets and related cytokines in infertile women undergoing in vitro fertilization before and after seminal plasma exposure
}

\author{
Marziyeh Azad', Sara Keshtgar', Bahia Namavar Jahromi', , Zahra Kanannejad', Behrouz Gharesi-Fard 1,4 \\ Departments of 'Immunology, ${ }^{2}$ Physiology, ${ }^{3}$ Obstetrics and Gynecology, and ${ }^{4}$ Infertility Research Center, Shiraz University of Medical Sciences, Shiraz, Iran
}

Objective: In vitro fertilization (IVF) is a well-known method for the treatment of infertility. The present study aimed to compare the differences between infertile women with successful and unsuccessful IVF outcomes regarding the expression of Thelper (Th) cell transcription factors and a group of related cytokines before and after exposure to their husbands' seminal plasma.

Methods: This study was performed on 19 couples with unexplained infertility undergoing IVF treatment. Among the studied group, nine and 10 couples had successful and unsuccessful IVF outcomes, respectively. This study was carried out using real-time polymerase chain reaction.

Results: Before seminal plasma exposure, the expression levels of T-bet $(p<0.007)$, interferon- $\gamma(p=0.013)$, and tumor necrosis factor (TNF)- $a$ $(p=0.017)$ were higher in the infertile women with IVF failure than in those with successful IVF outcomes, while those of GATA3 $(p<0.001)$, Foxp3 $(p=0.001)$, and interleukin (IL)-35 ( $p<0.003)$ were lower. After seminal exposure, the expression of T-bet $(p=0.02)$, Rorc $(p<0.001)$, TNF- $\alpha(p=0.001)$, Foxp3 $(p=0.02)$, and interferon- $\gamma(p=0.001)$ increased in the unsuccessful IVF group, while the expression of Foxp3 $(p=0.02)$, Rorc $(p<0.001)$, IL-23 ( $p=0.04)$, IL-17 ( $p=0.02)$, IL-6 ( $p<0.001)$, transforming growth factor- $\beta(p=0.01)$, and IL-35 $(p<0.001)$ increased in the successful IVF group.

Conclusion: In summary, IVF failure was associated with imbalanced Th1/Th2/Th17/Treg responses. Moreover, our results show that seminal plasma might have a positive effect on IVF outcomes via changes in peripheral blood T cell subsets.

Keywords: Cytokine; In vitro fertilization; T helper cell; Transcription factor

\section{Introduction}

Infertility has been defined as the failure to conceive successfully after 1 year of unprotected intercourse. This condition affects approximately $7 \%-15 \%$ of couples of reproductive age [1]. Many factors, such as endocrine and immunological factors, might predispose

Received: Jun 7, 2017 · Revised: Aug 4, 2017· Accepted: Oct 5, 2017 Corresponding author: Behrouz Gharesi-Fard

Department of Immunology, Shiraz University of Medical Sciences, Shiraz 71345-1798, Iran

Tel: +98-71-32351575 Fax:+98-71-32351575 E-mail: gharesifb@sums.ac.ir

*This study was extracted from the MSc thesis written by Marziyeh Azad and financially supported by the Shiraz University of Medical Sciences, Iran (No. 93-6979).

This is an Open Access article distributed under the terms of the Creative Commons Attribution Non-Commercial License (http://creativecommons.org/licenses/by-nc/4.0/) which permits unrestricted non-commercial use, distribution, and reproduction in any medium, provided the original work is properly cited. a couple to infertility. Some infertile couples are classified as having unexplained infertility because the reasons for their infertility remain unknown. Some evidence has been found to indicate that the immune system is involved in unexplained infertility [2].

In vitro fertilization (IVF) is widely used to treat infertility, but the outcomes of IVF are diverse and unpredictable. There is increasing evidence of the role of the maternal immune system in infertility [24]. Both humoral and cell-mediated immunity have been reported as possible mechanisms of implantation failure $[5,6]$. Regulation of the maternal immune response has also been found to be critical for implantation and the achievement of a successful pregnancy. In this regard, homeostasis between the type 1 helper T cell (Th1) and type 2 helper T cell (Th2) responses and Th1/Th2 polarization in implantation have been studied for many years. Recent studies have reported that fine-tuned balance and coordination among different subsets of 
Thelper cells contributed to fertility and the success of pregnancy [7]. Distinct $T$ cell subsets secrete various cytokines that play different roles in a complex regulatory pathway. Th1 cells produce inflammatory cytokines, such as interferon (IFN)- $\gamma$ and IFN- $a$, thereby promoting cell-mediated immunity. In contrast, Th2 cells are involved in humoral immunity by producing interleukin (IL)-4, IL-5, and IL-13 [7].

For many years, the Th1/Th2 paradigm has been used as a framework for predicting pregnancy outcomes. Several studies have confirmed that successful pregnancy is associated with a predominant Th2-type immunity, while Th1-type immunity is associated with pregnancy-related disorders, such as recurrent pregnancy loss (RPL) [8]. After the discovery of newer T helper subsets, we now speak of the Th1/Th2/Th17/Treg paradigm, rather than the Th1/Th2 paradigm [7]. T helper 17 cell (Th17) is a novel subset of T cells that develop from naive $C D 4^{+} T$ cells and secrete IL-17A, IL-17F, and IL-22. Rorc is the major transcriptional factor for Th17 development in humans and mice $[9,10]$. Th17 cells are directly involved in chronic inflammatory processes by secreting IL-17, which recruits neutrophils to tissues through the induction of granulocyte colony-stimulating factor and IL-8 [11]. Few published studies have focused on the role of Th17 cells in women with RPL. The results of those studies showed that Th17 cells and their related cytokines, including IL-23 and IL-17, were elevated in women with RPL compared to those with normal pregnancies [12]. Increased numbers of $\mathrm{IL}-17^{+} \mathrm{T}$ cells and a higher ratio of Th17 to $\mathrm{CD}^{+} / \mathrm{CD} 25^{+}$regulatory $\mathrm{T}$ cells (Treg) have also been shown to be associated with pregnancy loss [13].

The main types of $T$ cells generated in the periphery from naive $\mathrm{CD}^{+} \mathrm{T}$ cells under the influence of transforming growth factor (TGF)- $\beta$ are Treg. Foxp3 has been identified as a major transcription factor for the generation and development of Treg [12]. In mice and humans, Treg increase during pregnancy and play a key role in protecting the fetus from the adverse effects of maternal immune responses against the semi-allograft fetus [14]. In line with the important role of Treg in pregnancy, several studies have shown that the number of Treg was lower in the peripheral blood or decidua of women with RPL [14].

The successful implantation and maintenance of a normal pregnancy requires cellular and molecular changes within the uterine environment during the preimplantation period $[15,16]$. In reproduction, seminal plasma (SP) is considered to be means for the transport of spermatozoa to the cervix and uterus. In addition, previous studies have reported that SP provides a rich environment for successful implantation [17]. Several molecules with immunological activity have been shown to be present in SP, including TGF- $\beta$, IFN- $\gamma$, prostaglandin $\mathrm{E}_{2}$, tumor necrosis factor (TNF)- $\alpha$, IL-1 $\beta$, IL-6, IL-8, IL-10, and IL-12 [18]. These molecules are able to induce a classic inflammatory response within the female reproductive tract after insemination, which is nec- essary for successful implantation [19]. It has also been shown that semen can influence the production of certain cytokines, such as IL10 [20]. Studies using mouse models have demonstrated that SP increased the regulatory $T$ cell pool and induced tolerance to paternal alloantigens $[21,22]$. The elevated synthesis of IL-1 $\beta, I L-6$, and leukemia inhibitory factor by endometrial epithelial cells after culture with SP has also been document [23]. Overall, it seems that seminal fluid induces important immune reactions in the female reproductive tract that result in embryo development, implantation, and trophoblast invasion. Moreover, activated lymphocytes following insemination might mediate the maternal tolerance of the conceptus at the implantation site [24]. In this regard, some clinical evidence has confirmed that intercourse or artificial insemination during IVF cycles promotes embryo development and improves pregnancy rates [25]. Therefore, for a successful implantation and pregnancy, it is not only necessary for a sufficient number of spermatozoa to reach the oocyte in the oviduct, but also for the semen to regulate immune responses to ensure a successful outcome [25-27].

The present study aimed to investigate peripheral blood Th1, Th2, Th17, and Treg subsets and their related cytokines in infertile women who were candidates for IVF. Considering the importance of semen in the development of immune responses during successful implantation and pregnancy, another goal of the study was to investigate the effects of human semen on the production and differentiation of $T$ cell subsets and their related cytokines in these women.

\section{Methods}

\section{Study population}

This study was performed in 19 infertile couples who were candidates for IVF. Within this sample, 10 and nine couples had failed (unsuccessful) and successful IVF outcomes, respectively. A successful outcome was defined as a positive pregnancy test, while an unsuccessful outcome was defined as a negative human chorionic gonadotropin test after embryo transfer. It should be noted that all the couples had unexplained infertility and no history of recurrent miscarriage, autoimmune diseases, or immunodeficiency. Infertility was diagnosed by the same gynecologist and infertility specialist in couples who had engaged in regular unprotected intercourse for at least 1 year. The women were all undergoing IVF treatment at the IVF Center at Mother and Child Hospital, Shiraz University of Medical Sciences, Shiraz, Iran. The women did not engage in sexual intercourse for at least 2 weeks before IVF, and were not exposed to SP before or during the IVF procedure. Informed consent was obtained from the participants, and the study was approved by the Local Ethics Committee of Shiraz University of Medical Sciences for the use of blood samples and seminal fluid (No. 93-6979). 


\section{Semen collection and processing}

SP was obtained from all 19 partners of the infertile women. First, the semen samples were collected by masturbation using sterile containers. All semen samples had a normal sperm count, motility, and morphology according to the 2010 World Health Organization semen analysis criteria (5th edition) and were free of lymphoid cells (checked by light microscopy). The semen samples were allowed to liquefy for 30 minutes, and the SP was separated by centrifugation of the ejaculates at 1,200 rpm at room temperature for 10 minutes. The second round of centrifugation was performed at 10,000 rpm for 12 minutes to completely remove all the spermatozoa. Finally, SP samples were collected from the supernatants and stored at $-20^{\circ} \mathrm{C}$ until co-culture with peripheral blood mononuclear cells (PBMCs).

\section{Isolation and culture of PBMCs}

Fresh PBMCs were isolated from the infertile women using Ficoll density gradient centrifugation. Mononuclear cells were suspended in RPMI 1640 medium (Shellmax, Taizhou, China) supplemented with $10 \%$ fetal bovine serum (Shellmax) and 1\% penicillin-streptomycin (Invitrogen, Carlsbad, CA, USA). Subsequently, $1 \times 10^{6}$ separated PBMCs were stored in liquid nitrogen in order to extract the total RNA for the analysis of gene expression using real-time polymerase chain reaction (RT-PCR). Moreover, $1 \times 10^{6}$ separated PBMCs were used for co-culture with SP. At first, the separated cells were supplemented with $10 \%$ fetal bovine serum and $1 \%$ penicillin/streptomycin in RPMI medium and were then cultured with or without $0.33 \% \mathrm{SP}$ for 24,48 , and 72 hours at $37^{\circ} \mathrm{C}$ and $5 \% \mathrm{CO}_{2}$. At each time point, the viability of the cells was assessed by trypan blue staining. Wells with $>90 \%$ viability were harvested and stored in liquid nitrogen until the extraction of total RNA.

\section{RNA extraction}

RNA was extracted from a pellet of approximately $1 \times 10^{6}$ PBMCs using a total RNA extraction kit (Jena Bioscience, Jena, Germany) following the manufacturer's instructions. The final RNA pellet was dissolved in $25 \mu \mathrm{L}$ of diethyl pyrocarbonate (DEPC)-treated water. The concentration of the extracted RNA was determined using a Nano Drop instrument (NanoDrop 2000c; Thermo Scientific, Waltham, MA, USA). Agarose gel electrophoresis was used to check the purity and quality of the extracted mRNA. All extracted RNA was immediately converted to cDNA after extraction.

\section{5. cDNA synthesis}

CDNA was synthesized from $700 \mathrm{ng}$ of total RNA using an easy cDNA synthesis kit (Cinnagen, Tehran, Iran) based on the manufacturer's instructions. The final volume of the CDNA synthesis reaction was $20 \mu \mathrm{L}$, including $2 \mu \mathrm{L}$ of RNA, $2 \mu \mathrm{L}$ of a random hexamer and oligo-dT, $6 \mu \mathrm{L}$ of DEPC-treated water, and $10 \mu \mathrm{L}$ of $\times 2$ reverse transcriptase reaction buffer. The synthesis tube was then incubated at $65^{\circ} \mathrm{C}$ for 5 minutes, $25^{\circ} \mathrm{C}$ for 10 minutes, $47^{\circ} \mathrm{C}$ for 60 minutes, and $70^{\circ} \mathrm{C}$ for 10 minutes. The quality of all synthetic CDNA was checked by PCR.

\section{Quantitative RT-PCR}

The mRNA levels of all cytokines and $T$ cell transcription factors (TNF- $\alpha$, IFN- $\gamma$, IL-17A, IL-23, IL-31, IL-6, IL-35, TGF- $\beta$, Rorc, Foxp3, GATA3, and T-bet) were measured by means of RT-PCR using the SYBR Green method (GeNet Bio, Daejeon, Korea) in a StepOne RealTime PCR System (Applied Biosystems, Foster City, CA, USA). Briefly, 20 pmol of each primer (Table 1), $2 \mu \mathrm{L}$ of target CDNA (selected after checking and normalizing the CT values), and $10 \mu \mathrm{L}$ of the SYBR Green cocktail were used for amplification. The final volume of each

Table 1. Primer sequences used for real-time polymerase chain reaction

\begin{tabular}{|c|c|c|}
\hline Gene & Forward primer & Reverse primer \\
\hline IFN- $-\gamma$ & СTAATTATTCGGTAACTGACTTGA & ACAGTTCAGCCATCACTTGGA \\
\hline IL-6 & AAATTCGGTACATCCTCGAC & ССTCTTTGCTGCTTTCACAC \\
\hline TNF- $a$ & GCCTGCTGCACTTTGGAGTG & TCGGGGTTCGAGAAGATGAT \\
\hline TGF- $\beta$ & AAATTGAGGGCTTTCGCCTTA & TGAACCCGTTGATGTCCACTT \\
\hline IL-35 (EBI3) & ССТTCACCACTCCCAAAAC & GGCTTGATGATGTGCTCTG \\
\hline IL-35 (P35) & ССТTCACCACTCCCAAAAC & TGTCTGGCCTTCTGGAGCAT \\
\hline IL-23 & CCAAGGACTCAGGGACAACT & ATCAGGGAGCAGAGAAGGCT \\
\hline IL-31 & GATGATGTACAGAAAATAGTCGAGGAATT & СТTСТСТTССТССАСАТСТTTCAАA \\
\hline GATA3 & AGATGGCACGGGACACTACCT & GCCTTCGCTTGGGCTTAAT \\
\hline Foxp3 & CACCTGGAAGAACGCCATCC & CTCATCCACGGTGGTCCACACAG \\
\hline Rorc & CCCACAGATTTTGCAAGGGA & GCTGAGAAGGACAGGGAGCC \\
\hline T-bet & AACACAGGAGCGCACTGGAT & TGGAGGGACTGGAGCACAAT \\
\hline IL-17 & GGAAGAAACAACGATGAC & GATTCCTGCCTTCACTAT \\
\hline $18 \mathrm{~s}$ & CTCAACACGGGAAACCTCAC & AAATCGCTCCACCAACTAAGAA \\
\hline
\end{tabular}

IFN, interferon; IL, interleukin; TNF, tumor necrosis factor; TGF, transforming growth factor; GATA3, Th2 cell transcription factor; Foxp3, Treg cell transcription factor; Rorc, Th17 cell transcription factor; T-bet, Th1 cell transcription factor. 
reaction was $20 \mu \mathrm{L}$. The quantitative $\mathrm{PCR}(\mathrm{Q}-\mathrm{PCR})$ reactions were carried out as follows: initial denaturation at $95^{\circ} \mathrm{C}$ for 30 seconds followed by 40 cycles of denaturation at $95^{\circ} \mathrm{C}$ for 5 seconds and annealing and amplification at $60^{\circ} \mathrm{C}$ for 34 seconds. The comparative CT and fold differentiation ( $2^{-\Delta \Delta C T}$ ) methods were used to quantify target gene expression. Furthermore, the calibrator sample and 18s rRNA (a control housekeeping gene) were used to minimize the variation and to normalize the Q-PCR, respectively.

\section{Statistical analysis}

The Mann-Whitney test was used for the quantitative analysis of the Q-PCR results using GraphPad Prism ver. 5.0 (GraphPad Software Inc., San Diego, CA, USA), and $p$-values less than 0.05 were considered to indicate statistical significance. In addition, $\Delta C T$ values were used to compare gene expression in the cases and controls, and $2^{-\Delta \Delta C T}$ was used for relative gene expression analysis.

\section{Results}

\section{Differences between the women with successful and unsuccessful IVF outcomes regarding the expression of T cell transcription factors}

The expression of the T-bet transcription factor was significantly higher in the women with IVF failure than in those with successful IVF outcomes $(p=0.007)$. However, the expression of the GATA3 and Foxp3 transcription factors was significantly lower in the women with IVF failure than in those with successful IVF outcomes $(p<0.001$ and $p=0.001$, respectively) (Figure 1). No significant differences were found between the two groups regarding the expression of the Rorc transcription factor.

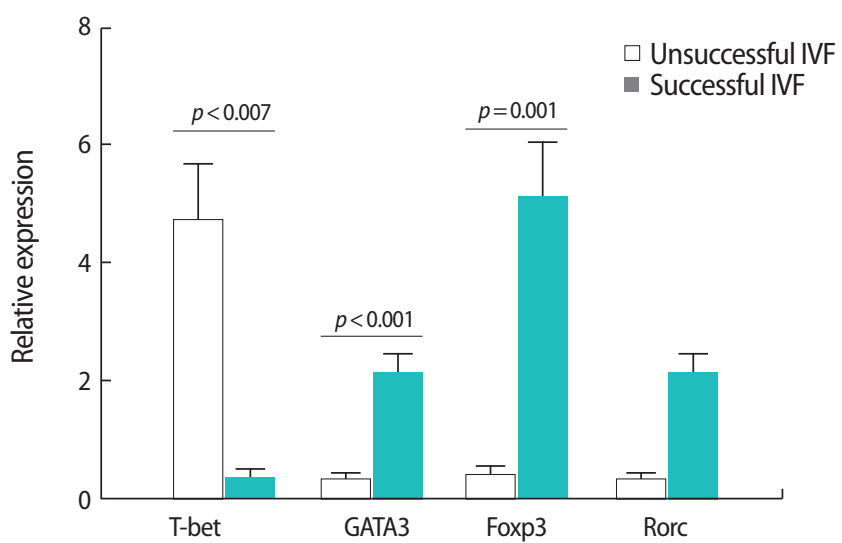

Figure 1. Comparison of the relative expression levels of the studied T cell transcription factors in peripheral blood samples from infertile women with successful $(n=9)$ and unsuccessful $(n=10)$ in vitro fertilization (IVF) outcomes. The data were analyzed by the Mann-Whitney test.

\section{Differences between the women with successful and unsuccessful IVF outcomes regarding the expressions of cytokines associated with Th1, Th2, Th17, and Treg subsets}

As shown in Figure 2, the expression of IFN- $\gamma(p=0.013)$ and TNF- $a$ $(p=0.017)$ was upregulated in the women with IVF failure in comparison to the successful IVF group. However, the expression of both chains of the IL-35 cytokine was downregulated in the women with IVF failure compared to the successful IVF group $(p=0.035$ and $p=0.03$ for the EBI3 and P35 subunits, respectively). Nevertheless, no significant differences were observed between the two groups regarding other cytokines ( $p=0.7$ for both IL-23 and IL-17, $p=0.09$ for both IL- 6 and TGF- $\beta$, and $p=0.4$ for IL-31).

The ratio of the expression of cytokines to the expression of tran-

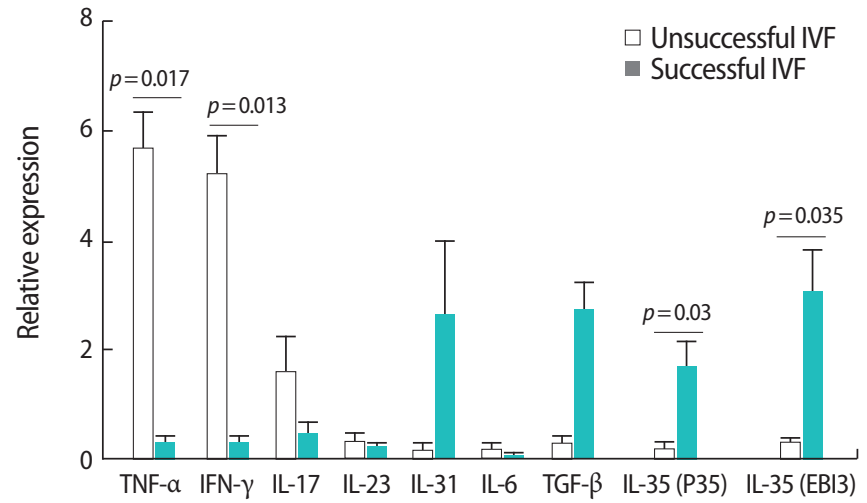

Figure 2. Comparison of the relative expression levels of the studied cytokines in peripheral blood samples from infertile women with successful $(n=9)$ and unsuccessful $(n=10)$ in vitro fertilization (IVF) outcomes. The data were analyzed by the Mann-Whitney test. TNF, tumor necrosis factor; IFN, interferon; IL, interleukin; TGF, transforming growth factor.

Table 2. Comparison of the ratio of the expression of transcription factors to that of cytokines in women with successful and unsuccessful IVF outcomes

\begin{tabular}{lccc}
\hline Expression ratio & Successful IVF & Unsuccessful IVF & $p$-value ${ }^{\text {a) }}$ \\
\hline T-bet/GATA3 & $0.88 \pm 0.10$ & $1.39 \pm 0.06$ & 0.007 \\
TNF-a/IL-31 & $0.90 \pm 0.04$ & $1.24 \pm 0.10$ & 0.005 \\
TNF-a/GATA3 & $0.94 \pm 0.01$ & $1.13 \pm 0.05$ & $<0.001$ \\
IFN- $/$ GATA3 & $0.87 \pm 0.05$ & $1.29 \pm 0.04$ & 0.001 \\
Foxp3/Rorc & $0.95 \pm 0.10$ & $0.74 \pm 0.04$ & 0.020 \\
Foxp3/IL-17 & $1.24 \pm 0.01$ & $0.85 \pm 0.01$ & 0.020 \\
IL-35/IL-17 & $1.20 \pm 0.01$ & $0.84 \pm 0.05$ & 0.007 \\
IL-35/TNF-a & $1.09 \pm 0.04$ & $0.73 \pm 0.04$ & 0.002 \\
IL-35/IFN- $\gamma$ & $1.10 \pm 0.05$ & $0.83 \pm 0.05$ & 0.010 \\
\hline
\end{tabular}

Values are presented as mean \pm standard error.

IVF, in vitro fertilization; TNF, tumor necrosis factor; IL, interleukin; IFN, interferon.

a) Exact $p$-values calculated by the Mann-Whitney test. The $p$-values $<0.05$ were considered to indicate statistical significance. 
scription factors was also investigated. The significant results are presented in Table 2. While the ratios of T-bet to GATA3, TNF- $a$ to IL-31, TNF- $a$ to GATA3, and IFN- $\gamma$ to GATA3 were all higher in women with IVF failure than in the successful IVF group, the ratios of Foxp3 to Rorc, Foxp3 to IL-17, IL-35 to TNF- $a$, IL-35 to IFN- $\gamma$, and IL-35 to IL-17 were significantly lower in the IVF failure group.

\section{Difference in the expression of $\mathrm{T}$ cell transcription factors before and after SP exposure}

In both groups of women, no significant changes were seen in GATA3 expression after SP exposure. However, both groups expressed more GATA3 after SP exposure. T-bet expression significantly increased after SP exposure only in women with unsuccessful IVF outcomes $(p=0.02)$. The expression of the Rorc transcription factor
A

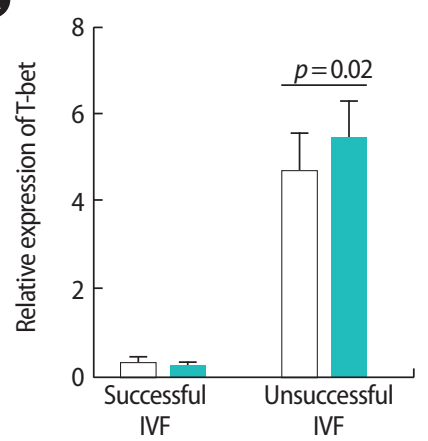

B

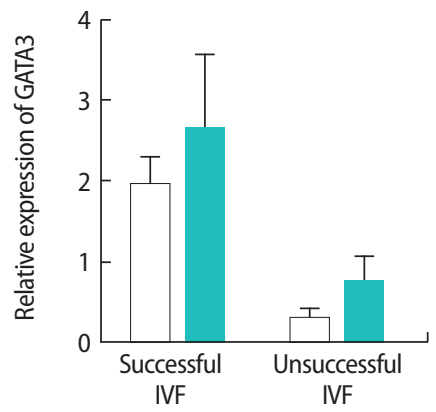

$\square$ Before SP exposure $\square$ After SP exposure

Figure 3. Comparison of the relative expression levels of T cell transcription factors of (A) T-bet, (B) GATA3, (C) Foxp3, (D) Rorc before and after seminal plasma (SP) exposure in peripheral blood samples from infertile women with successful $(n=9)$ and unsuccessful $(n=10)$ in vitro fertilization (IVF) outcomes. The data were analyzed by the Mann-Whitney test, and $p$-values $<0.05$ were considered to indicate statistical significance.

A

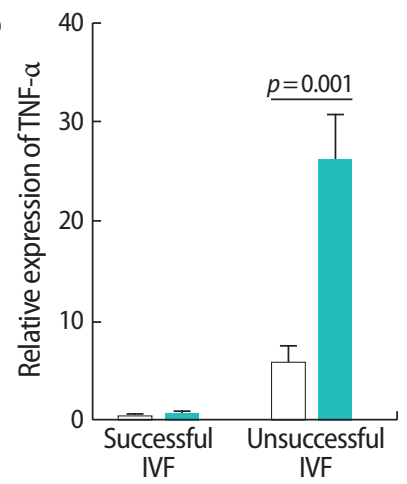

(D)

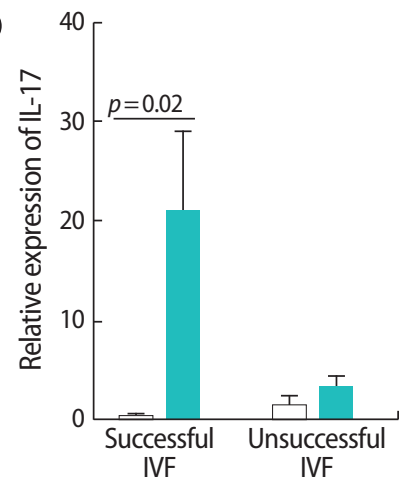

B

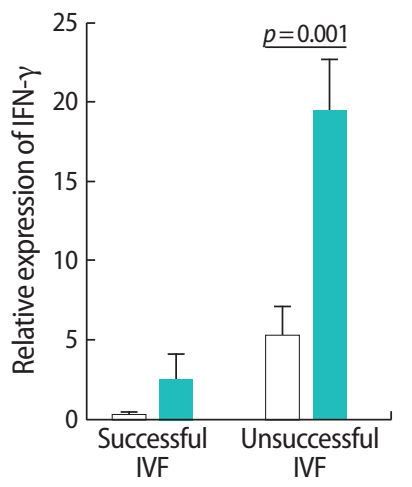

E

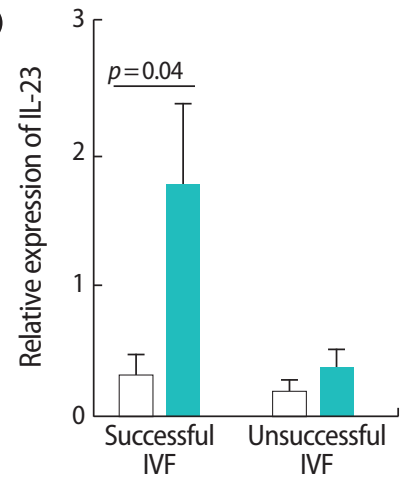

C

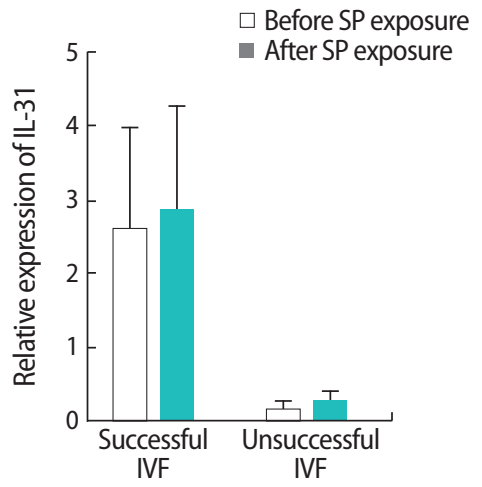

F

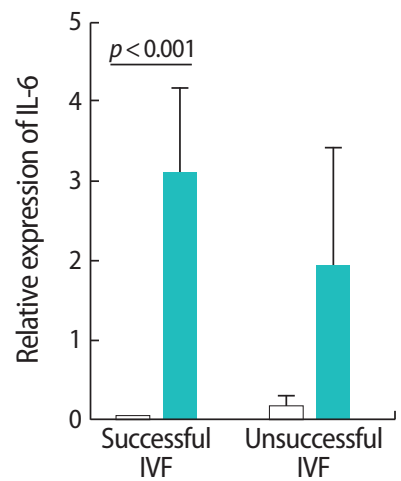

Figure 4. Comparison of the relative expression levels of (A) tumor necrosis factor (TNF)- $a$, (B) interferon (IFN)- - , (C) interleukin (IL)-31, (D) IL-17, (E) IL-23, (F) IL-6 before and after seminal plasma (SP) exposure in peripheral blood samples from infertile women with successful and unsuccessful in vitro fertilization (IVF) outcomes. The data were analyzed by the Mann-Whitney test, and $p$-values $<0.05$ were considered to indicate statistical significance. 
A

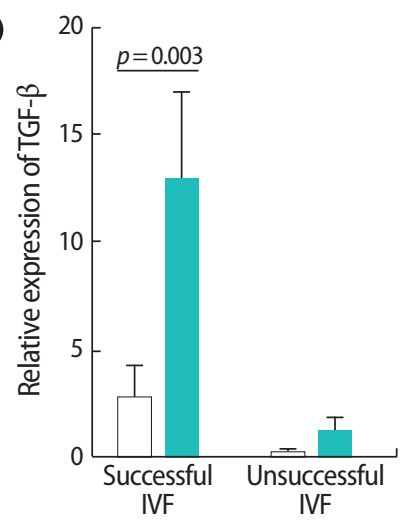

B

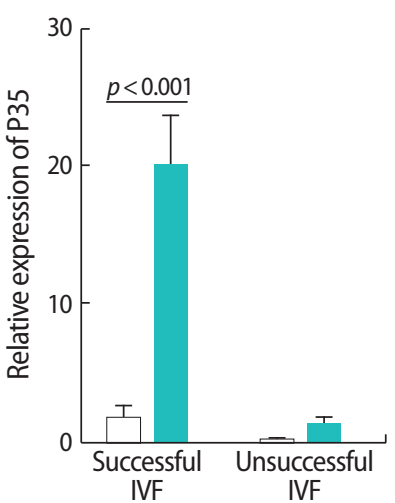

C

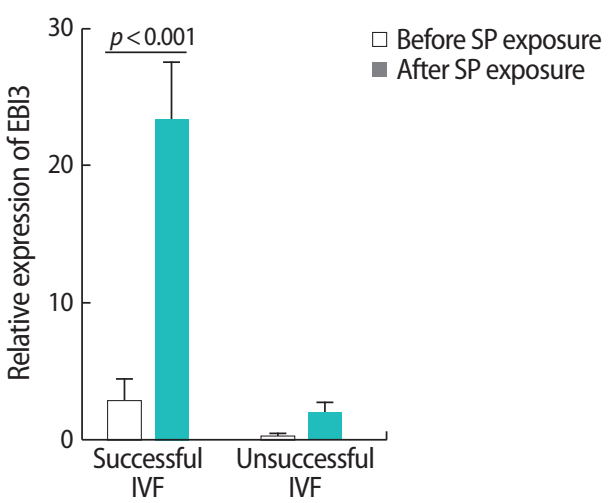

Figure 5. Comparison of the relative expression levels of (A) transforming growth factor (TGF)- $\beta,(B, C)$ interleukin (IL)-35 (P35 and EBI3) before and after seminal plasma (SP) exposure in peripheral blood samples from infertile women with successful and unsuccessful in vitro fertilization (IVF) outcomes. The data were analyzed by the Mann-Whitney test, and $p$-values $<0.05$ were considered to indicate statistical significance.

Table 3. Comparison of the ratios of the expression of transcription factors to that of cytokines after SP exposure in women with successful and unsuccessful IVF outcomes

\begin{tabular}{lccc}
\hline Ratio & Successful IVF & Unsuccessful IVF & $p$-value ${ }^{\text {a) }}$ \\
\hline IFN- $/ / L-31$ & $0.08 \pm 0.04$ & $2.60 \pm 0.10$ & 0.005 \\
TNF-a/IL-31 & $0.82 \pm 0.05$ & $1.35 \pm 0.08$ & 0.001 \\
Foxp3/Rorc & $1.41 \pm 0.12$ & $0.92 \pm 0.05$ & 0.006 \\
IL-35/TNF-a & $1.20 \pm 0.06$ & $0.57 \pm 0.10$ & 0.003 \\
IL-35/IFN- $\gamma$ & $1.20 \pm 0.07$ & $0.63 \pm 0.03$ & 0.003 \\
\hline
\end{tabular}

Values are presented as mean \pm standard error.

$\mathrm{SP}$, seminal plasma; IVF, in vitro fertilization; IFN, interferon; IL, interleukin; TNF, tumor necrosis factor.

a) Exact $p$-values calculated by the Mann-Whitney test. The $p$-values $<0.05$ were considered to indicate statistical significance.

was also upregulated after SP exposure in both groups $(p<0.001)$. Foxp3 expression also showed significant upregulation after SP exposure in both groups $(p=0.02)$ (Figure 3 ).

\section{Differences in the expression of cytokines associated with Th1, Th2, Th17, and Treg responses after SP exposure}

Our investigation of cytokine expression indicated that after SP exposure, the expression of TNF- $a$ was upregulated in the women with unsuccessful IVF outcomes $(p=0.001)$. In the women with successful outcomes, however, the expression of IL-17 ( $p=0.02)$, IL-23 ( $p=0.04)$, and IL-6 ( $p<0.001)$ significantly increased. After SP exposure, no significant changes were observed in IL-31 expression in either group (Figure 4). In the women with successful outcomes, the expression of all Treg-associated cytokines (i.e., TGF- $\beta[p=0.003]$ and IL-35 [ $p<0.001$ for $\mathrm{P} 35$ and $p<0.001$ for EBI3]), showed significant upregulation after SP exposure (Figure 5).

The results of the comparison between successful and unsuccessful IVF groups regarding the ratio of the expression of cytokines after SP exposure are presented in Table 3. The ratios of IFN- $\gamma$ to IL-31 and TNF- $a$ to IL-31 were significantly higher $(p=0.005$ and $p=0.001$, respectively) in the women with IVF failure than in those with successful IVF outcomes, while the ratios of Foxp3 to Rorc, IL-35 to TNF- $a$, and IL-35 to IFN- $\gamma$ were significantly lower ( $p=0.006, p=0.003$, and $p=0.003$, respectively) (Table 3 ). Moreover, a comparison of the relative expression of transcription factors and their related cytokines before and after SP exposure only found two significant changes: the ratios of IFN- $y$ to IL-31 and Foxp3 to Rorc were upregulated in the unsuccessful and successful groups, respectively ( $p=0.005$ for both comparisons, not shown in a table).

\section{Correlations between $T$ cell transcription factors and their associated cytokines after SP exposure}

After SP exposure, a negative correlation was found between the expression of Foxp3 and Rorc ( $r=-0.65, p=0.038$ ) (Figure 6A), IL-35 (EBI3) and TNF- $\alpha(r=-0.75, p=0.072)$ (Figure 6B), and TGF- $\beta$ and Tbet $(r=-0.59, p=0.006)$ (Figure $6 C)$ in the women with successful IVF outcomes. In contrast, in the women with unsuccessful IVF outcomes, the expression of the Rorc transcription factor was positively correlated with TNF- $\alpha(r=0.75, p=0.01)$ (Figure 7A) and the expression of T-bet was correlated to IFN- $\gamma(r=0.81, p=0.005)$ (Figure 7B) before SP exposure. After SP exposure, however, the expression of IFN- $\gamma$ showed a positive correlation with TNF- $\alpha(r=0.69, p=0.03)$ (Figure 8A) and the expression of IL-17 was correlated to TNF-a $(r=0.65, p=0.041)$ (Figure 8B) and IFN- $\gamma(r=0.75, p=0.02)$ (Figure $8 C)$ in the women with unsuccessful IVF outcomes.

\section{Discussion}

This study compared the expression of the transcription factors of Th cell subsets and their related cytokines in infertile women with 

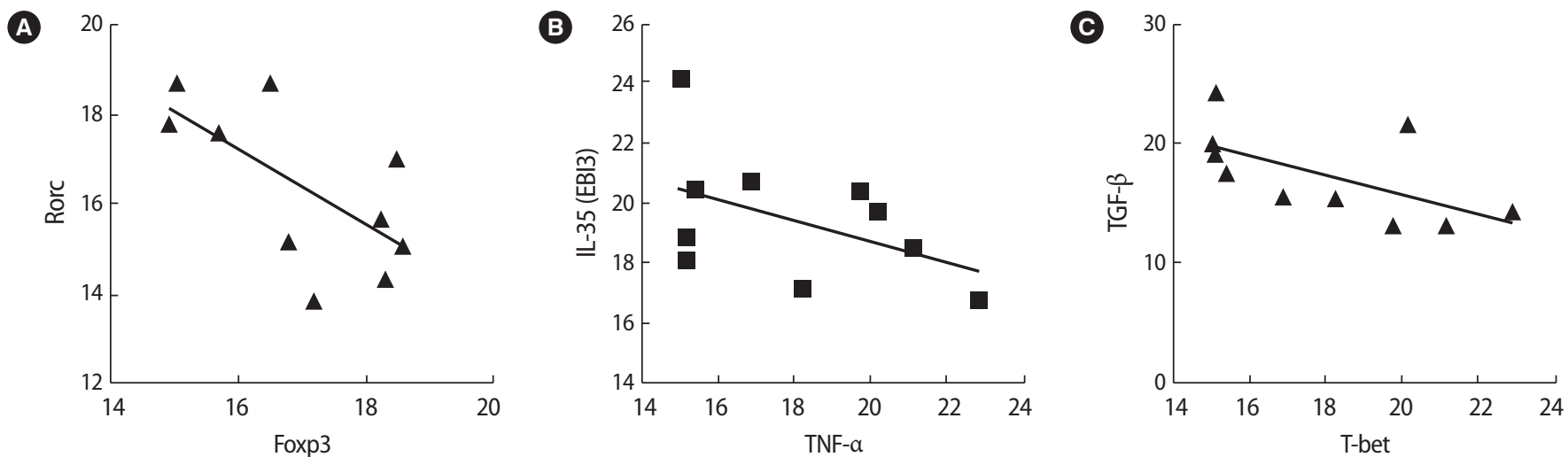

Figure 6. Correlations between the expression of T cell transcription factors and their associated cytokines in infertile women with successful in vitro fertilization outcomes before seminal plasma exposure: (A) Rorc and Foxp3, (B) interleukin (IL)-35 (EBI3) and tumor necrosis factor (TNF)-a, (C) transforming growth factor (TGF)- $\beta$ and T-bet.
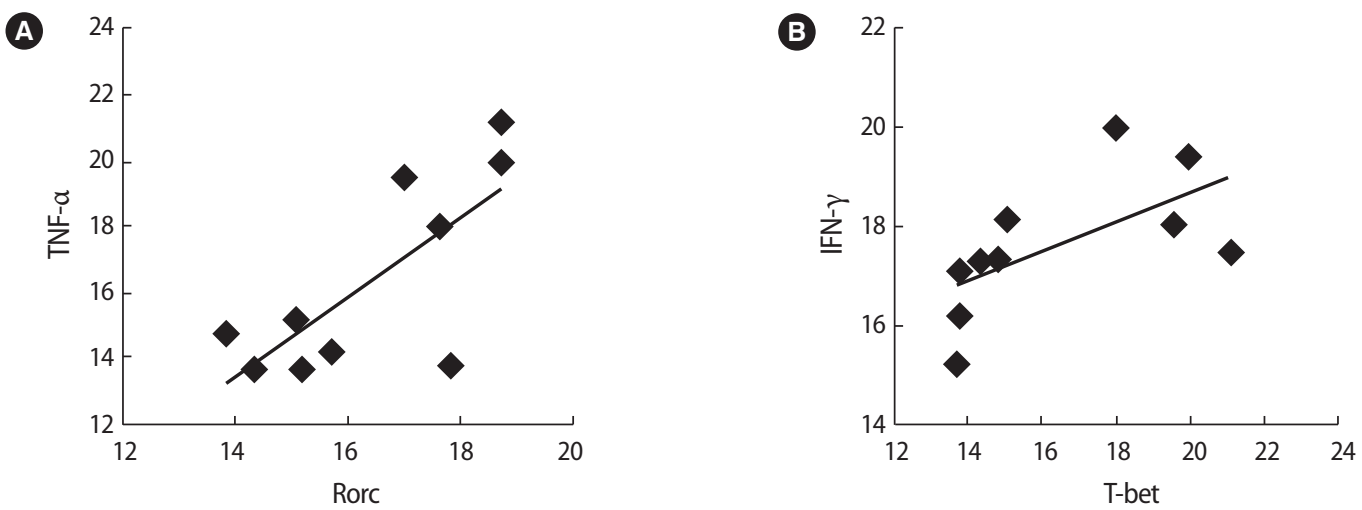

Figure 7. Correlations between the expression of T cell transcription factors and their associated cytokines in infertile women with unsuccessful in vitro fertilization outcomes before seminal plasma exposure: (A) tumor necrosis factor (TNF-a) and Rorc, (B) interferon (IFN)- $\gamma$ and T-bet.
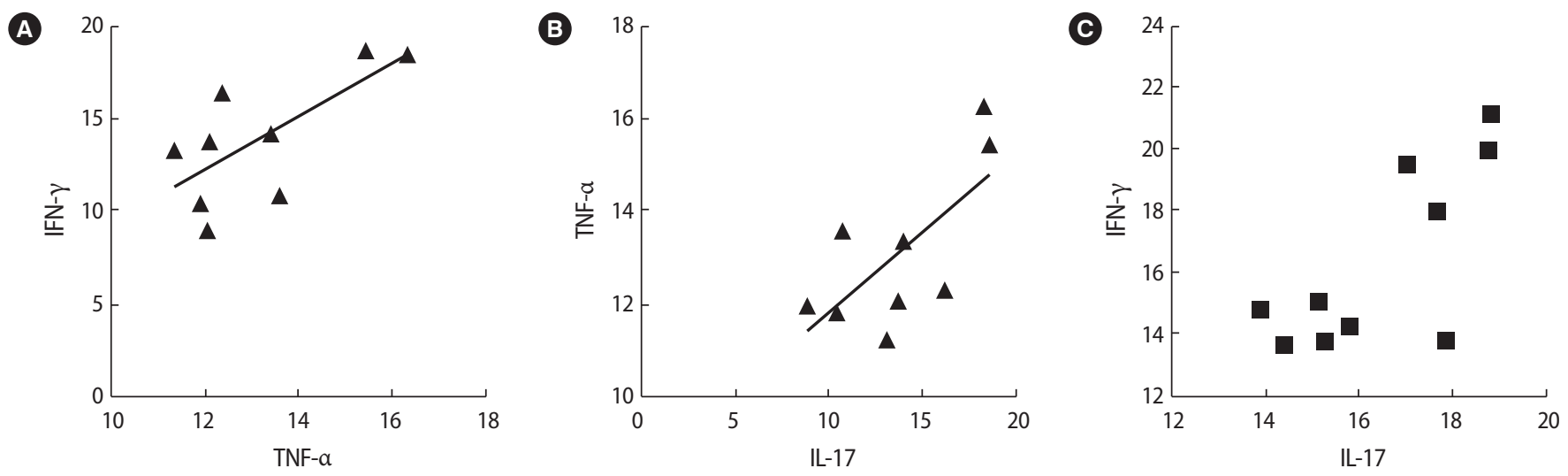

Figure 8. Correlations between the expression of T cell transcription factors and their associated cytokines in infertile women with unsuccessful in vitro fertilization outcomes after seminal plasma exposure: (A) interferon (IFN)- $\gamma$ and tumor necrosis factor (TNF- $a$ ) (B) TNF- $a$ and interleukin (IL)-17, (C) IFN- $\gamma$ and IL-17.

successful and unsuccessful IVF outcomes before and after SP exposure. Previous studies have documented that a group of infertility patterns might be associated with inappropriate activity of the immune system. Indeed, several studies have indicated that effector mechanisms of cellular immunity played an important role in the etiology of unexplained infertility [2-4]. Moreover, it is generally agreed that pregnancy is associated with a predominance of Th2-type immunity, while an increased Th1 immune response is associated with 
RPL and multiple implantation failures [28-31]. In line with previous studies, the results of the present research showed a Th1 bias regarding immune responses in the infertile women with IVF failure. A dominant Th1 response in women with IVF failure was also demonstrated by the increased expression of T-bet and its associated Th1 cytokines IFN- $\gamma$ and TNF- $a$. In contrast, Th2-dominant responses in the infertile women with successful IVF outcomes were associated with the upregulation of GATA3 expression. Moreover, in agreement with previous findings, our results showed that infertile women with IVF failure had higher ratios of T-bet to GATA3, TNF- $\alpha$ to GATA3, IFN- $\gamma$ to GATA3, and TNF-a to IL-31 than the successful IVF group. Therefore, an adequate balance of Th1 and Th2 immunity that is slightly shifted towards Th2-type immunity may be effective for achieving a successful IVF outcome. However, overstimulation of the Th1 or Th2 immune response may reduce the likelihood of a successful IVF outcome. In recent years, the important role of the Th17/Treg subsets in pregnancy outcomes has been highlighted and, as a result, the Th1/Th2 paradigm has been expanded toward the Th1/Th2/Th17/Treg paradigm. Th17 and Treg cells have opposite effects, as Th17 plays an important role in the inflammatory immune response, but Treg cells are a potent suppressor of inflammation [14,32]. Both Treg and Th17 cells are involved in the establishment and maintenance of pregnancy as regulator and effector cells, respectively. Several studies have suggested that an imbalance between regulatory and effector cells might lead to implantation failure and many other pregnancy-related disorders [7]. Treg have recently been considered as key players in protecting the conceptus from the adverse effects of alloreactive responses that are mediated by the mother's immune system [33]. Furthermore, the number of Treg has been reported to diminish in spontaneous abortion compared to elective abortion [34]. The results of the present work indicated that the Treg transcription factor (Foxp3) and IL-35 expression were significantly higher in the women with successful IVF outcomes than in the IVF failure group. IL-35 is an anti-inflammatory cytokine that belongs to the IL-12 family and is mainly produced by Treg. IL-35 exerts a suppressive activity on the immune system through the expansion of Treg and the inhibition of Th17 cell development [35,36]. A few studies have reported that IL-35 significantly increased in normal pregnancies and decreased in recurrent spontaneous abortion [37]. Moreover, the ratio of IL-35 to IL-17 has been reported to be lower in infertile women than in healthy ones [38,39]. As in other published studies, our results showed an increase in the Treg to Th1 ratio (IL-35 to INF- $\gamma$ and IL-35 to TNF- $\alpha$ ) and negative correlations between IL-35 and TNF- $a$, T-bet, and TGF- $\beta$ in the women with successful IVF outcomes compared to those with IVF failure. This result underscores the importance of the regulation of immune responses, as well as inflammatory responses, at the time of fertilization and implantation for the success of IVF. Supporting this hypoth- esis, our results indicated that in women with unsuccessful IVF outcomes, inflammatory responses were upregulated, while regulatory responses were diminished. Although our results showed no significant differences between the two studied groups regarding IL-17 production, the ratios of Treg/Th17 transcription factors to their related cytokines (Foxp3 to Rorc, Foxp3 to IL-17, and IL-35 to IL-17) were higher in the women with successful outcomes. Furthermore, a negative correlation was detected between Foxp3 and Rorc in the women with successful IVF outcomes. These results show that the inflammatory response by Th17 must be controlled by Treg cells in order to achieve successful fertilization and implantation.

In the second part of this study, for the first time, the effect of SP on the expression of $\mathrm{T}$ cell transcription factors and their related cytokines was investigated in infertile women with successful and unsuccessful IVF outcomes. In the successful IVF group, SP exposure increased the inflammatory response. In this group, along with an increase in inflammation, regulatory responses were also upregulated to control inflammation. In the women with IVF failure, although the inflammatory responses increased, the regulatory responses did not increase in parallel to control the adverse effects of inflammation. Thus, it seems that women with IVF failure are not able to control the inflammatory response and that semen exposure is not able to compensate for this problem. In line with this hypothesis, the findings of the current study demonstrated an increase in the expressions of Rorc, T-bet, IFN- $\gamma$, and TNF-a, but no overexpression of regulatory T cell cytokines, after SP exposure in patients with IVF failure. Therefore, in the unsuccessful group, SP might have exacerbated inflammation by increasing the expansion of Th1 and Th17 cells. On this basis, it can be concluded that women with IVF failure might have a defect in the induction of their Treg that cannot be compensated for by SP exposure. In a recently published paper, Robertson et al. [40] reviewed the effects of SP on the cervix, endometrium, immune responses, and outcomes of assisted reproductive technology and IVF. Seminal fluid was reported to play a role in embryo development, implantation, and trophoblast invasion [23]. Moreover, several previous studies have revealed the role of SP in feto-maternal tolerance and the generation of Treg $[41,42]$. Among the many proposed effects of semen, the induction of proinflammatory cytokines and chemokines, which are important for implantation, is well known [43]. In line with this important effect of SP, the results of the present study indicated that SP might increase the expression of inflammatory cytokines and inflammatory $T$ cell subsets. In both the successful and unsuccessful groups, SP exposure elevated the expression of inflammatory cytokines. Hence, it may be concluded that SP affects the success of implantation by priming the mother to accept the fetus. Furthermore, seminal fluid contains several potent immune suppressor agents that might induce the expansion of inducible Treg cells. Indeed, the 
most important pathway mediated by SP is the activation and expansion of maternal Treg [41].

Consistent with previous studies, the findings of the present study indicate that SP might induce Treg and their related cytokines. Interestingly, this effect was only observed in the women with successful IVF outcomes. In the women with unsuccessful outcomes, however, SP exposure increased Treg cytokines. Nonetheless, this increase was not significant and was not sufficient to control the inflammation. This discrepancy may have been due to a difference between the successful and unsuccessful groups regarding the composition of semen, including semen cytokines or Treg, and this possibility should be investigated further. Moreover, SP exposure might reduce the risk of complications of pregnancy, such as pre-eclampsia and effects on the offspring's metabolic phenotype [44]. Although no paper has been previously published with which we could compare our results directly, some studies have suggested that women with pregnancy disorders, such as RPL, had a proinflammatory tendency via Th1 and Th17 immunity and a decrease in immune regulatory function by Foxp $3^{+}$Treg [41]. In summary, the results of the present study indicate that IVF failure was associated with imbalanced Th1/Th2/Th17/ Treg responses. The results also showed that SP might have a positive effect on IVF outcomes via changes in peripheral blood T cell subsets.

\section{Conflict of interest}

No potential conflict of interest relevant to this article was reported.

\section{Acknowledgments}

The authors would like to thank Ms. Afsaneh Keivanshekouh at the Research Improvement Center of Shiraz University of Medical Sciences for improving the use of English in the manuscript.

\section{References}

1. Montoya JM, Bernal A, Borrero C. Diagnostics in assisted human reproduction. Reprod Biomed Online 2002;5:198-210.

2. Ray A, Shah A, Gudi A, Homburg R. Unexplained infertility: an update and review of practice. Reprod Biomed Online 2012;24: 591-602.

3. Akhter S, Alam H, Khanam NN, Zabin F. Characteristics of infertile couples. Mymensingh Med J 2011;20:121-7.

4. Matzuk MM, Lamb DJ. The biology of infertility: research advances and clinical challenges. Nat Med 2008;14:1197-213.

5. Anderson DJ, Hill JA. Cell-mediated immunity in infertility. Am J Reprod Immunol Microbiol 1988;17:22-30.

6. Carp HJ, Selmi C, Shoenfeld Y. The autoimmune bases of infertili- ty and pregnancy loss. J Autoimmun 2012;38:J266-74.

7. Saito $S$, Nakashima A, Shima T, Ito M. Th1/Th2/Th17 and regulatory T-cell paradigm in pregnancy. Am J Reprod Immunol 2010; 63:601-10.

8. Chaouat $\mathrm{G}$. The Th1/Th2 paradigm: still important in pregnancy? Semin Immunopathol 2007;29:95-113.

9. Crome SQ, Wang AY, Levings MK. Translational mini-review series on Th17 cells: function and regulation of human Thelper 17 cells in health and disease. Clin Exp Immunol 2010;159:109-19.

10. Nakashima A, Ito M, Yoneda S, Shiozaki A, Hidaka T, Saito S. Circulating and decidual Th17 cell levels in healthy pregnancy. Am J Reprod Immunol 2010;63:104-9.

11. Kolls JK, Linden A. Interleukin-17 family members and inflammation. Immunity 2004;21:467-76.

12. Wang WJ, Hao CF, Yi-Lin, Yin GJ, Bao SH, Qiu LH, et al. Increased prevalence of T helper 17 (Th17) cells in peripheral blood and decidua in unexplained recurrent spontaneous abortion patients. J Reprod Immunol 2010;84:164-70.

13. Lee SK, Kim JY, Hur SE, Kim CJ, Na BJ, Lee M, et al. An imbalance in interleukin-17-producing $T$ and Foxp3+ regulatory $T$ cells in women with idiopathic recurrent pregnancy loss. Hum Reprod 2011;26:2964-71.

14. Saito S, Sasaki Y, Sakai M. CD4(+)CD25high regulatory T cells in human pregnancy. J Reprod Immunol 2005;65:111-20.

15. Robertson SA, Mau VJ, Hudson SN, Tremellen KP. Cytokine-leukocyte networks and the establishment of pregnancy. Am J Reprod Immunol 1997;37:438-42.

16. Black CA, Rohan LC, Cost M, Watkins SC, Draviam R, Alber S, et al. Vaginal mucosa serves as an inductive site for tolerance. J Immunol 2000;165:5077-83.

17. Crawford G, Ray A, Gudi A, Shah A, Homburg R. The role of seminal plasma for improved outcomes during in vitro fertilization treatment: review of the literature and meta-analysis. Hum Reprod Update 2015;21:275-84.

18. Maegawa M, Kamada M, Irahara M, Yamamoto S, Yoshikawa S, Kasai Y, et al. A repertoire of cytokines in human seminal plasma. J Reprod Immunol 2002;54:33-42.

19. Politch JA, Tucker L, Bowman FP, Anderson DJ. Concentrations and significance of cytokines and other immunologic factors in semen of healthy fertile men. Hum Reprod 2007;22:2928-35.

20. Jeremias J, Mockel S, Witkin SS. Human semen induces interleukin 10 and $70 \mathrm{kDa}$ heat shock protein gene transcription and inhibits interferon-gamma messenger RNA production in peripheral blood mononuclear cells. Mol Hum Reprod 1998;4:1084-8.

21. Robertson SA, Guerin LR, Bromfield JJ, Branson KM, Ahlstrom $A C$, Care AS. Seminal fluid drives expansion of the CD4+CD25+ T regulatory cell pool and induces tolerance to paternal alloanti- 
gens in mice. Biol Reprod 2009;80:1036-45.

22. Guerin LR, Moldenhauer LM, Prins JR, Bromfield JJ, Hayball JD, Robertson SA. Seminal fluid regulates accumulation of FOXP3+ regulatory $T$ cells in the preimplantation mouse uterus through expanding the FOXP3+ cell pool and CCL19-mediated recruitment. Biol Reprod 2011;85:397-408.

23. Gutsche $S$, von Wolff M, Strowitzki T, Thaler CJ. Seminal plasma induces mRNA expression of IL-1 1 beta, IL-6 and LIF in endometrial epithelial cells in vitro. Mol Hum Reprod 2003;9:785-91.

24. Johansson M, Bromfield JJ, Jasper MJ, Robertson SA. Semen activates the female immune response during early pregnancy in mice. Immunology 2004;112:290-300.

25. Tremellen KP, Valbuena D, Landeras J, Ballesteros A, Martinez J, Mendoza $S$, et al. The effect of intercourse on pregnancy rates during assisted human reproduction. Hum Reprod 2000;15: 2653-8.

26. Aflatoonian A, Ghandi S, Tabibnejad N. The effect of intercourse around embryo transfer on pregnancy rate in assisted reproductive technology cycles. Int J Fertil Steril 2009;4.

27. Fishel S, Webster J, Jackson P, Faratian B. Evaluation of high vaginal insemination at oocyte recovery in patients undergoing in vitro fertilization. Fertil Steril 1989;51:135-8.

28. Kwak-Kim JY, Chung-Bang HS, Ng SC, Ntrivalas El, Mangubat CP, Beaman KD, et al. Increased Thelper 1 cytokine responses by circulating $\mathrm{T}$ cells are present in women with recurrent pregnancy losses and in infertile women with multiple implantation failures after IVF. Hum Reprod 2003;18:767-73.

29. Raghupathy R. Th1-type immunity is incompatible with successful pregnancy. Immunol Today 1997;18:478-82.

30. Ng SC, Gilman-Sachs A, Thaker P, Beaman KD, Beer AE, Kwak-Kim J. Expression of intracellular Th1 and Th2 cytokines in women with recurrent spontaneous abortion, implantation failures after IVF/ET or normal pregnancy. Am J Reprod Immunol 2002;48:7786.

31. Ginsburg ES, Xiao L, Gargiulo AR, Kung FT, Politch JA, Schust DJ, et al. T-helper 2 and 3 type immunity to trophoblast in successful in vitro fertilization-embryo transfer. Fertil Steril 2005;83: 1659-64.

32. Lee SK, Kim JY, Lee M, Gilman-Sachs A, Kwak-Kim J. Th17 and regulatory $T$ cells in women with recurrent pregnancy loss. Am J Reprod Immunol 2012;67:311-8.
33. Sakaguchi S. Naturally arising Foxp3-expressing CD25+CD4+ regulatory $T$ cells in immunological tolerance to self and nonself. Nat Immunol 2005;6:345-52.

34. Sasaki Y, Sakai M, Miyazaki S, Higuma S, Shiozaki A, Saito S. Decidual and peripheral blood CD4+CD25+ regulatory T cells in early pregnancy subjects and spontaneous abortion cases. Mol Hum Reprod 2004;10:347-53.

35. Yue CY, Zhang B, Ying CM. Elevated serum level of IL-35 associated with the maintenance of maternal-fetal immune tolerance in normal pregnancy. PLoS One 2015;10:e0128219.

36. Whitehead GS, Wilson RH, Nakano K, Burch LH, Nakano H, Cook DN. IL-35 production by inducible costimulator (ICOS)-positive regulatory T cells reverses established IL-17-dependent allergic airways disease. J Allergy Clin Immunol 2012;129:207-15.e1-5.

37. Niedbala W, Wei XQ, Cai B, Hueber AJ, Leung BP, McInnes IB, et al. IL-35 is a novel cytokine with therapeutic effects against collagen-induced arthritis through the expansion of regulatory $T$ cells and suppression of Th17 cells. Eur J Immunol 2007;37:30219.

38. Ozkan ZS, Deveci D, Kumbak B, Simsek M, Ilhan F, Sekercioglu S, et al. What is the impact of Th1/Th2 ratio, SOCS3, IL17, and IL35 levels in unexplained infertility? J Reprod Immunol 2014;103:538.

39. Robertson SA, Sharkey DJ. Seminal fluid and fertility in women. Fertil Steril 2016;106:511-9.

40. Schjenken JE, Robertson SA. Seminal fluid signalling in the female reproductive tract: implications for reproductive success and offspring health. Adv Exp Med Biol 2015;868:127-58.

41. Robertson SA, Prins JR, Sharkey DJ, Moldenhauer LM. Seminal fluid and the generation of regulatory $T$ cells for embryo implantation. Am J Reprod Immunol 2013;69:315-30.

42. Sharkey DJ, Macpherson AM, Tremellen KP, Robertson SA. Seminal plasma differentially regulates inflammatory cytokine gene expression in human cervical and vaginal epithelial cells. Mol Hum Reprod 2007;13:491-501.

43. Hyde KJ, Schust DJ. Immunologic challenges of human reproduction: an evolving story. Fertil Steril 2016;106:499-510.

44. Bromfield JJ, Schjenken JE, Chin PY, Care AS, Jasper MJ, Robertson SA. Maternal tract factors contribute to paternal seminal fluid impact on metabolic phenotype in offspring. Proc Natl Acad Sci U S A 2014;111:2200-5. 